\title{
Field-based multiplex and quantitative assay platforms for diagnostics
}

\author{
Srivatsa Venkatasubbarao ${ }^{* a}$, Edward C. Dixon ${ }^{\mathrm{b}}$, Russell Chipman ${ }^{\mathrm{c}}$, Axel Scherer ${ }^{\mathrm{d}}$, Manal Beshay ${ }^{\mathrm{a}}$, \\ Lothar U. Kempen ${ }^{\mathrm{a}}$, Jai Ganesh Chandra Sekhar ${ }^{\mathrm{a}}$, Hong Yan ${ }^{\mathrm{b}}$, Ava Puccio ${ }^{\mathrm{b}}$, David Okonkwo ${ }^{\mathrm{b}}$, \\ Stephan McClain ${ }^{\mathrm{c}}$, Noah Gilbert ${ }^{\mathrm{c}}$, Saurabh Vyawahare ${ }^{\mathrm{d}}$ \\ ${ }^{a}$ Intelligent Optical Systems, Inc., Torrance, CA, USA 90505-5217 \\ ${ }^{\mathrm{b}}$ University of Pittsburgh, Pittsburgh, PA, USA 15213 \\ ${ }^{\mathrm{c}}$ University of Arizona, Tucson, AZ, USA 85721 \\ ${ }^{\mathrm{d}}$ California Institute of Technology, Pasadena, CA 91125
}

\begin{abstract}
The U.S. military has a continued interest in the development of handheld, field-usable sensors and test kits for a variety of diagnostic applications, such as traumatic brain injury (TBI) and infectious diseases. Field-use presents unique challenges for biosensor design, both for the readout unit and for the biological assay platform. We have developed robust biosensor devices that offer ultra-high sensitivity and also meet field-use needs. The systems under development include a multiplexed quantitative lateral flow test strip for TBI diagnostics, a field test kit for the diagnosis of pathogens endemic to the Middle East, and a microfluidic assay platform with a label-free reader for performing complex biological automated assays in the field.
\end{abstract}

Keywords: lateral flow immunoassay, dipstick assay, lateral flow test strip, field-based diagnostics, microfluidic chips, labelless detection, traumatic brain injury

\section{INTRODUCTION}

Advances in the fields of genomics and proteomics are offering a number of promising biomarkers that can aid in accurately predicting different diseases and health conditions. It is postulated that changes in the gene and protein expression levels can aid in the diagnosis of traumatic brain injury, the identification of chemical, biological or radiological exposures, organ damage, and failure of the immune system, and in the monitoring of physical stress and fatigue, epidemiological diseases, and other health conditions. To exploit the diagnostic potential of the biomarkers, it is necessary to build devices that can be used to assay these biomarkers in bodily fluids. Typically, multiple biomarkers need to be measured to accurately predict a particular health condition. Therefore, the diagnostic device must have the capability to detect/measure multiple biomarkers. In a clinical laboratory, complex automated instruments can be used to perform such assays. However, in a field setting, laboratory grade instruments are not appropriate because they: (1) are too complex to be useful for field use; (2) are too bulky to be carried; (3) require multiple steps to extract the sample and conduct tests; (4) require highly skilled personnel to run the instruments; (5) require labels to detect the biomarkers; (6) require multiple reagents to conduct the assays; and (7) are not reusable. Furthermore, many of these assays cannot withstand the conditions (temperature, humidity, etc.) present in the field. For all of these reasons, there is an immediate need to develop a simple, lightweight, handheld device that can be used in the field, by military personnel, for multiplexed detection of biomarkers.

A field-usable diagnostic device to detect and measure biomarkers must meet the following requirements: (1) the assay developed for the biomarkers should preferably be reagent free, or utilize minimal amounts of reagents, to expeditiously and effectively conduct assays in the field without having to transport a number of reagents; (2) the device should detect/measure many biomarkers simultaneously in a single testing; (3) the device must be compact, lightweight, batterypowered, and miniaturized for handheld testing by personnel; (4) the sample collection and processing steps must be simple and automated to be helpful for field use; (5) the software used to run the device must be simple, and must be able to store data from previous measurements so that the trends of different biomarkers can be compared for

\footnotetext{
*srao@intopsys.com; phone 1424263 6344; fax 1310530 7417; intopsys.com
}

Sensing Technologies for Global Health, Military Medicine, Disaster Response, and Environmental Monitoring; and Biometric Technology for Human Identification VIII, eds. Southern, Montgomery, Taylor, Weigl, Vijaya Kumar, Prabhakar, Ross, Proc. of SPIE Vol. 8029, 80290P - (c) 2011 SPIE · CCC code: 0277-786X/11/\$18 - doi: 10.1117/12.886661 
longitudinal studies; and (6) since most of the biological elements are easily denatured by extremes of humidity and temperature, processes should be developed to stabilize the biological elements so that they can withstand these extremes.

The lateral flow test strip is an attractive sensing format for field applications because the required reagents are present on the test strip, and the sample and reagents are transported, by capillary action, across the test strip, and therefore no pumps and valves are required. These attributes of the lateral flow test strip have popularized its use. The sensitivity of conventional test strips, however, is moderate, and the quantification of analytes and multiplexed measurements are difficult. The ability to measure multiple biomarkers quantifiably, at high sensitivity, on a single test strip, is necessary.

An alternative technology that is attractive for field-based diagnostics would be a microfluidics assay platform. Microfluidics-based assays offer the promise of highly multiplexed measurements, precise control of sample volumes, and versatility in the manipulation of samples. Professor Axel Scherer's group at Caltech has developed a multi-layer soft lithography-based miniaturized microfluidic chip that consists of hundreds of microfluidic channels, valves, and pumps to transport nanoliter volumes of samples to the sensing areas. The microfluidic chip design consists of patterned channels (control layer), above the microfluidics layer, that deflect air pressure to membranes that function as valves and pumps. We report on the utilization of inexpensive and high sensitivity cameras, and the recent availability of miniature pumps and solenoid valves that can be used to pressurize the control layer, to create a field-usable device that meets military needs.

\section{TECHNOLOGY}

\subsection{Fieldable microfluidic assay readers}

The biomarker reader is designed, based on evanescent imaging ellipsometry, ${ }^{[1]}$ to measure the effect that the binding of biomolecules to the microarray surface has on the polarization state of the reflected light. The polarization state of the light is defined by the phase and amplitude relationships between two components. The component having the electric field in the plane of incidence is known as the $p$-polarized light, and the component having the electric field normal to the plane of incidence is called the $s$-polarized light. In general, the reflection of light from an optical thin film causes a relative change in the phase and amplitude of the $p$ and $s$ polarized light. The change in phase difference between $p$ and $s$ components is characterized by the angle $\Delta$, which is known as the retardance. The change in the amplitude ratio between $p$ and $s$ components is characterized by $\operatorname{Tan}(\psi)$. These definitions lead us to the fundamental equation of ellipsometry,

$$
\tan \Psi \exp (i \Delta)=\frac{r_{p}}{r_{s}}
$$

where $r_{p}$ and $r_{s}$ are the reflection coefficients for $p$-polarized light and $s$-polarized light, respectively. The change in the amplitude ratio between $p$ and $s$ components can also be characterized by diattenuation, which is defined as

$$
D=\operatorname{Cos}[2 \Psi]=\frac{\left|r_{s}\right|^{2}-\left|r_{p}\right|^{2}}{\left|r_{s}\right|^{2}+\left|r_{p}\right|^{2}} .
$$

A schematic diagram of the imaging ellipsometer is shown in Figure 1. In this configuration, the incident angle $\theta_{0}$ is larger than the critical angle required for Total Internal Reflection (TIR) from a glass prism surface in contact with a biomolecular film. The polarization state change is due to the TIR from the biomolecular film and the glass prism surface. If all the materials in the measurement sample are dielectric, only retardance $\Delta$ occurs at the TIR interface. Since the magnitude of the retardance $\Delta$ depends on the thickness and the refractive index of the film, the measurement of $\Delta$ will provide information about the optical parameters of the sample film. Furthermore, if absorbing materials are used in the sample diattenuation $D$ will also couple into the measurement. 


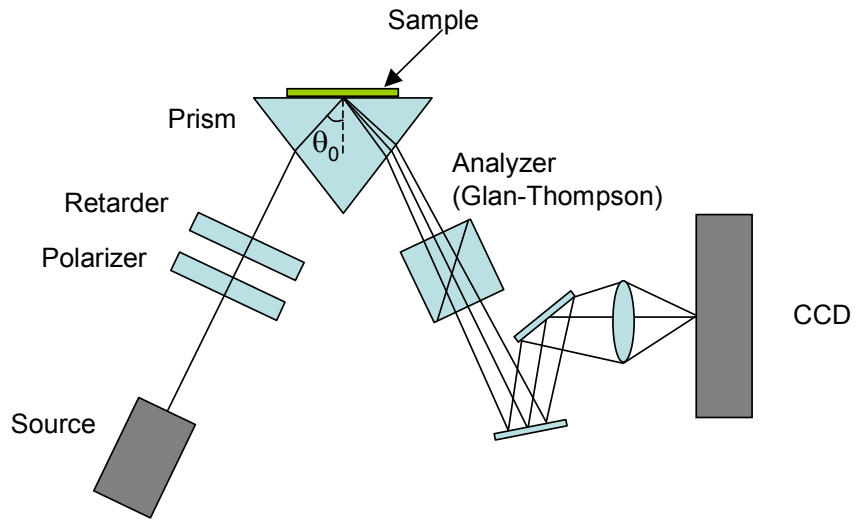

Figure 1 Principle of imaging ellipsometer microarray reader. Polarized light illuminates the microarray surface and is totally internally reflected. The changes in the retardance and diattenuation of the reflected beam are measured and related to the properties of the microarray surface.

The measurement principles of the imaging ellipsometer can be fully explained with the help of Mueller matrix calculus. ${ }^{[2]}$ Here, Jones matrix calculus is employed to provide a brief introduction of how the detection unit works.

As shown in Figure 1, the incident monochromatic beam is collimated and transmitted through a linear polarizer and a linear retarder (quarter wave plate). The azimuthal orientations of the polarizer and the quarter wave plate determine the relative amplitudes and phase difference between the $p$ and $s$ components of the beam incident upon the substrate. These orientations are adjusted in such a way that the amplitudes of the $p$ and $s$ components are equal, and the difference in phase compensates for the retardance that results from the TIR from the hypotenuse surface of the glass prism. Therefore, the beam reflected off the glass-air interface is linearly polarized at $45^{\circ}$ to the incident plane. It can be written in the form of a Jones vector as

$$
\frac{1}{2}\left(\begin{array}{l}
1 \\
1
\end{array}\right) \text {. }
$$

This Jones vector is not normalized. It has a modulus of 0.5 , because the polarizer only transmits half of the total power of the unpolarized incident beam.

The reflected beam is then transmitted by an analyzer to a telephoto lens and a CCD array. The analyzer is oriented at $135^{\circ}$. It can be written in terms of a Jones matrix as

$$
\left(\begin{array}{cc}
\frac{1}{2} & -\frac{1}{2} \\
-\frac{1}{2} & \frac{1}{2}
\end{array}\right) .
$$

If there were no sample film on the hypotenuse surface of the glass prism, in the ideal condition, the reflected beam would be completely extinguished by the analyzer,

$$
\left(\begin{array}{cc}
\frac{1}{2} & -\frac{1}{2} \\
-\frac{1}{2} & \frac{1}{2}
\end{array}\right) \cdot \frac{1}{2}\left(\begin{array}{l}
1 \\
1
\end{array}\right)=\left(\begin{array}{l}
0 \\
0
\end{array}\right) .
$$

However, by taking into account the retardance deviation $\Delta$ and diattenuation $\mathrm{D}$ introduced by the sample film on top of the glass surface, the left hand side of Equation 3-5 can be written in terms of the combination of two Jones matrices as

$$
\left(\begin{array}{cc}
1 & 0 \\
0 & e^{i \Delta}
\end{array}\right) \cdot\left(\begin{array}{cc}
\left|r_{s}\right| & 0 \\
0 & \left|r_{p}\right|
\end{array}\right) .
$$

The beam reflected by the sample surface is not a linearly polarized beam. Its polarization state can be represented as 


$$
\left(\begin{array}{cc}
1 & 0 \\
0 & e^{i \Delta}
\end{array}\right) \cdot\left(\begin{array}{cc}
\left|r_{s}\right| & 0 \\
0 & \left|r_{p}\right|
\end{array}\right) \cdot \frac{1}{2}\left(\begin{array}{l}
1 \\
1
\end{array}\right)=\frac{1}{2}\left(\begin{array}{c}
\left|r_{s}\right| \\
\left|r_{p}\right| e^{i \Delta}
\end{array}\right)
$$

Therefore, the beam will not be completely extinguished by the analyzer oriented at $135^{\circ}$. The polarization state of the transmitted light can be represented as

$$
\left(\begin{array}{cc}
\frac{1}{2} & -\frac{1}{2} \\
-\frac{1}{2} & \frac{1}{2}
\end{array}\right) \cdot \frac{1}{2}\left(\begin{array}{c}
\left|r_{s}\right| \\
\left|r_{p}\right| e^{i \Delta}
\end{array}\right)=\frac{\left|r_{s}\right|-\left|r_{p}\right| e^{i \Delta}}{2 \sqrt{2}} \frac{1}{\sqrt{2}}\left(\begin{array}{c}
1 \\
-1
\end{array}\right)
$$

After being transmitted by the analyzer oriented at $135^{\circ}$, the light is collected by a lens and forms an image of the sample surface on the CCD array. The intensity of the image can be written as

$I_{\text {out }}=\left|\frac{\left|r_{s}\right|-\left|r_{p}\right| e^{i \Delta}}{2 \sqrt{2}}\right|^{2}=\frac{\left|r_{s}\right|^{2}+\left|r_{p}\right|^{2}-2\left|r_{s}\right| \cdot\left|r_{p}\right| \cdot \operatorname{Cos}[\Delta]}{8}=\frac{\left(\left|r_{s}\right|-\left|r_{p}\right|\right)^{2}}{8}+\frac{1}{2}\left|r_{s}\right| \cdot\left|r_{p}\right| \cdot \operatorname{Sin}^{2}\left[\frac{\Delta}{2}\right]$.

Since the thickness of the sample is much smaller than the optical wavelength (thickness of sample is less than $10 \mathrm{~nm}$ and the wavelength of incident light is $520 \mathrm{~nm}$ ), we can use the following approximation to simplify the equation of the image intensity:

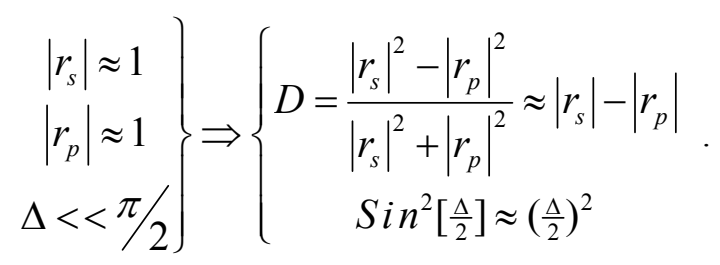

After the simplification, the image intensity can be written as

$$
I_{\text {out }}=\frac{\left(\left|r_{s}\right|-\left|r_{p}\right|\right)^{2}}{8}+\frac{1}{2}\left|r_{s}\right| \cdot\left|r_{p}\right| \cdot \operatorname{Sin}^{2}\left[\frac{\Delta}{2}\right] \approx \frac{D^{2}}{8}+\frac{1}{2} \operatorname{Sin}^{2}\left[\frac{\Delta}{2}\right] \approx \frac{D^{2}+\Delta^{2}}{8} .
$$

Therefore, in the microarray reader system, the relationship between $(\Delta, \mathrm{D})$ and the resulting image intensity $I_{\text {out }}$ is given by:

$$
I_{\text {out }} \approx \frac{D^{2}}{8}+\frac{1}{2} \operatorname{Sin}^{2}\left[\frac{\Delta}{2}\right] \approx \frac{D^{2}+\Delta^{2}}{8} \text { where } \Delta<<\pi / 2 \text { and } D<<1 .
$$

In summary, spatial variations in $\Delta$ and $D$ that occur due to the biomolecular layer result in the measured image intensity distribution. When the retardance deviation $\Delta$ and the diattenuation $D$ introduced by the sample film are small, the image intensity $I_{\text {out }}$ of the microarray reader is proportional to $\Delta^{2}+D^{2}$. Since the variation of $\Delta$ and $D$ in the 2D space are associated with the spatial variations in thickness $t$ or the refractive index $n$ of the sample film, the image intensity can provide useful information about the optical parameters of the sample film.

For a fully dielectric film, the contribution from diattenuation changes (D) is minimal and can be ignored. The intensity is then proportional to the square of the retardance changes. The calibration is based on the following calculations, where

$$
I=c \cdot \Delta^{2} \text {. }
$$

For extremely thin thickness changes, the Drude approximation can be used. According to the Drude approximation, ${ }^{[3,4]}$ the retardance deviation $\Delta$ is proportional to the thickness variation $t$ of the extremely thin film. Thus, the calibration equation (Eq. 13) can be changed into:

$$
I=c \cdot t^{2}
$$


The prototype microfluidic biomarker reader developed at IOS represents a technological breakthrough in evanescent imaging ellipsometric approach. To our knowledge, there are no commercial devices that have a similar performance or form factor. Previous efforts by our collaborator, Professor Axel Scherer, involved the miniaturization of the microfluidic chips. However, air delivery to operate the embedded microfluidic pumps and valves, as well as fluid delivery to these chips, has been based on large, laboratory-based devices. We have successfully completed the development of a handheld system with self-contained units for the transport of all fluids within the portable microfluidic unit. This represents a technological and engineering breakthrough with applications in many different commercial markets (Figure 2).

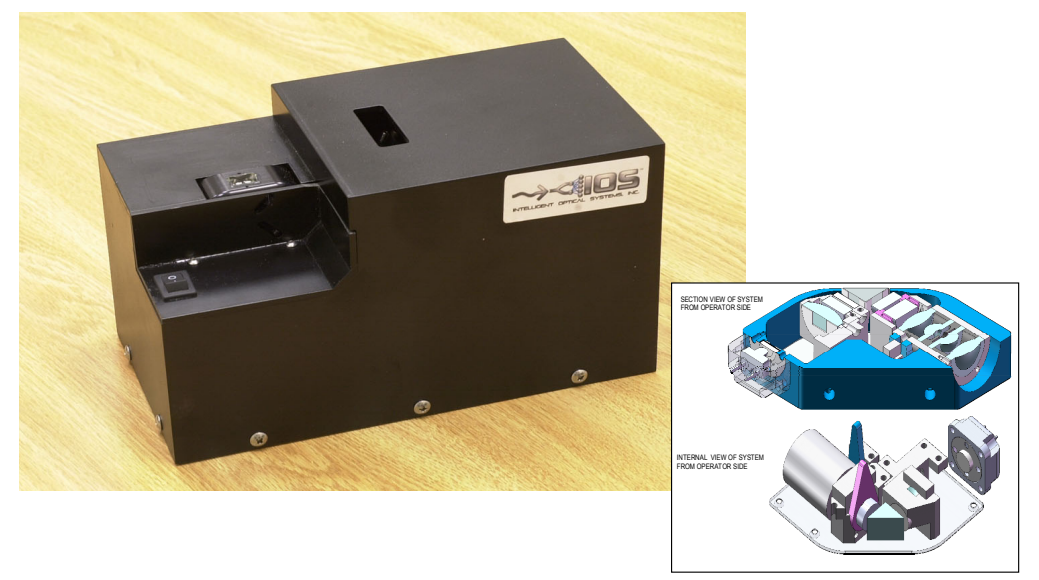

(a)

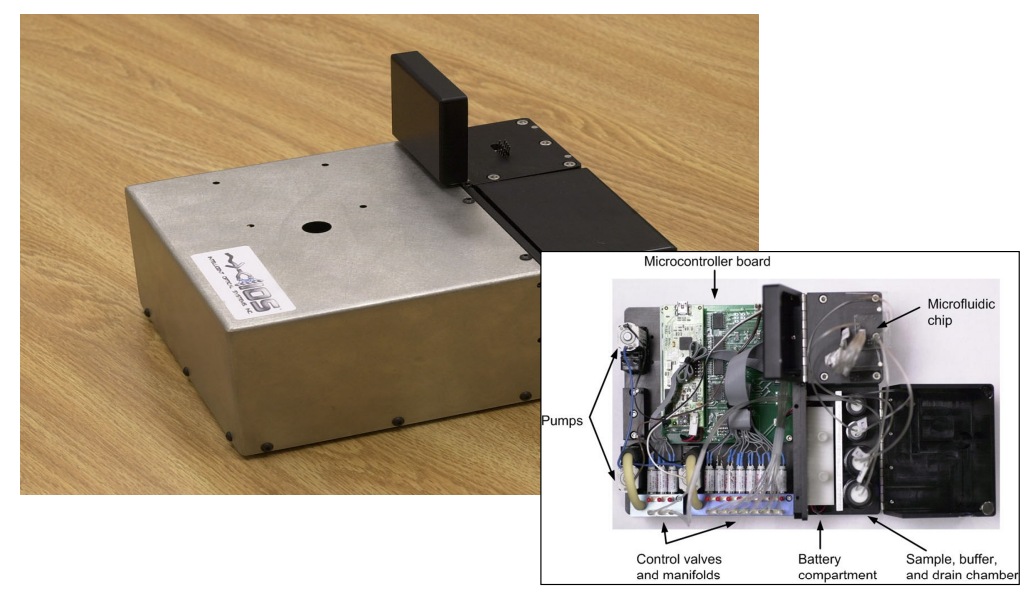

(b) 

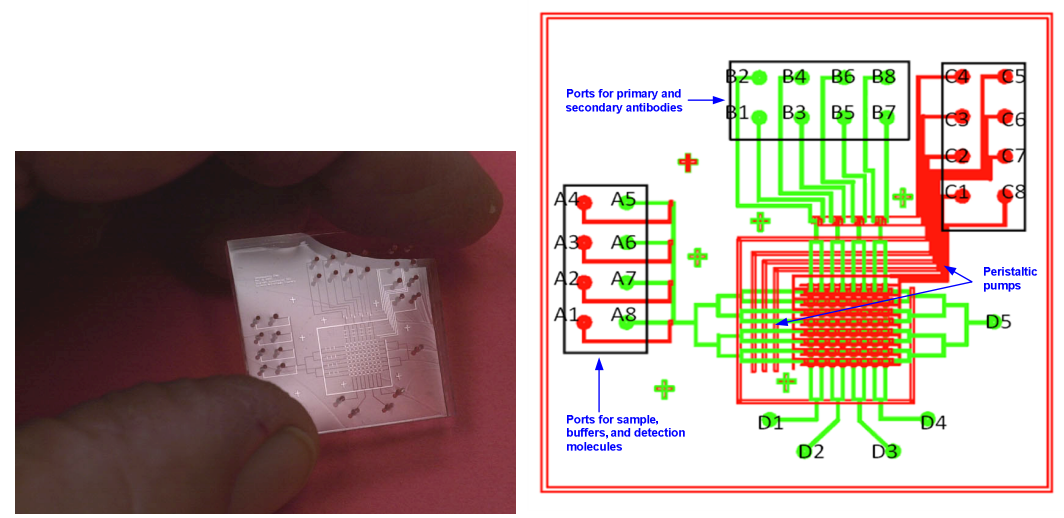

(c)

Figure 2 Hardware/software developed under this contract was delivered to the U.S. Air Force: (a) labelless microfluidic evanescent field-usable imaging ellipsometry reader; (b) field-usable microfluidic system to transport reagents through the microfluidic chip; (c) microfluidic chips developed using soft lithography techniques.

Soft lithography is a microfabrication technique in which a soft polymer such as PDMS is cast onto a mold containing a microfabricated relief. This technique has been used to create channels, valves, and pumps. This allows large-scale integration of microfluidic devices. Samples of nanoliter and picoliter volumes can be controlled with reproducibility and unprecedented control.

The fabrication of the microfluidic sensor chips starts with a mold made in a silicon wafer - usually using photolithography. Next an elastomeric polymer, such as polydimethoxysilane (PDMS), is poured on top of the mold, and is then cured using UV. On curing, the elastomeric polymer layer is peeled off. This leaves an imprint of the mold topography on the surface of the polymer. Similarly, multiple layers of these elastomers with different patterns are bonded to each other to form a complete microfluidic device.

A basic microfluidic chip contains two elastomer layers. One layer contains channels for flowing liquids (flow layer), and the other layer contains channels that when pressurized with air or nitrogen function as valves and pumps for the flow channels (control layer) as shown in Figure 2. Bonding of different layers is done thermally or using oxygen plasma. A slight difference between curing agent present in different layers is sufficient to allow two layers to bond.

A microfluidic valve is created when two channels are cross-separated by a thin elastomeric membrane (Figure 3). The membrane is not shown explicitly in Figure 3. The space between the two channels is where the membrane is placed. In our approach, the flow layer of the chip is made very thin. This becomes the membrane (i.e., there will be a thin layer of PDMS above the channels in the flow layer). This thin layer separates the flow and control lines. The "d" in Figure 4 represents the membrane thickness. When the control channel is pressurized with air, the thin membrane separating the two channels deflects into the flow channels creating a complete seal. 


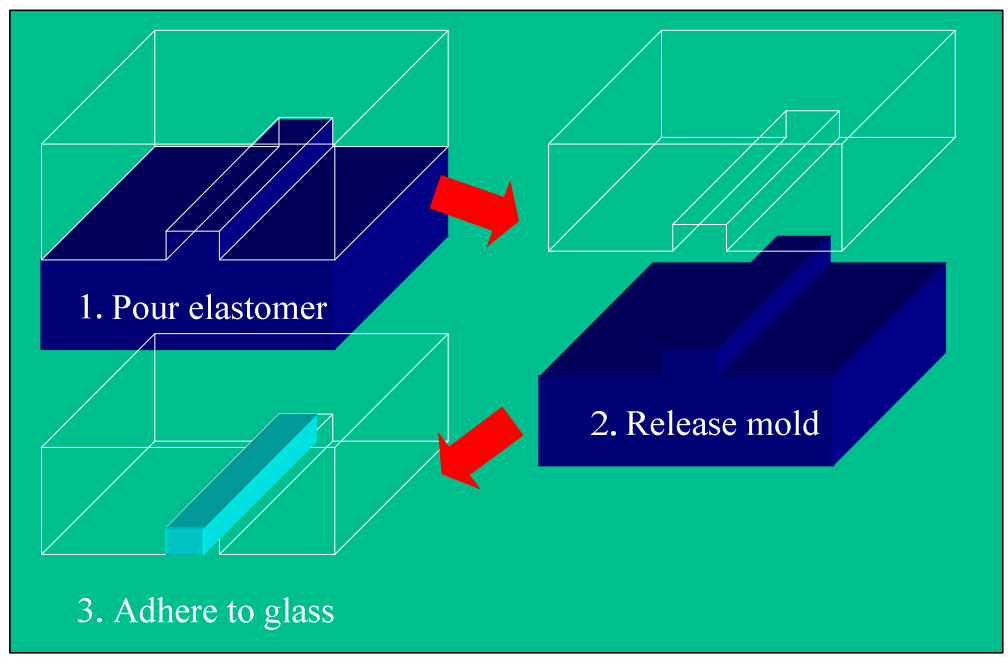

Figure 3 Fabrication of microfluidic channels using soft lithography.

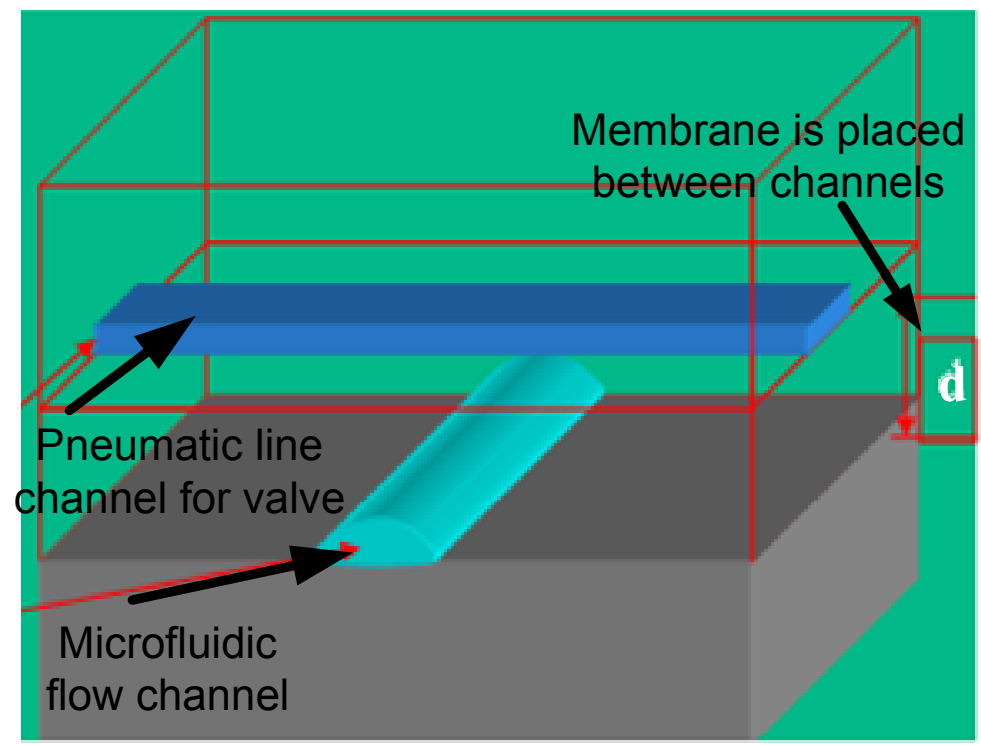

Figure 4 Fabrication of valves ( $d=$ membrane thickness).

Pressurizing one channel causes the membrane to deflect and block the other channel, creating a valve. A peristaltic pump is created when three valves in a row are operated in a sequence. This type of combination of valves and pumps can create fairly sophisticated circuits. These chips are extremely small (about one inch square), and can contain hundreds of valves and peristaltic pumps.

\subsection{Multiplexed quantitative lateral flow test strips}

Gold, silver, carbon, latex, colored dyes, enzymes, and fluorescent dyes have been used as labels in lateral flow test strips. Each of these labels possesses certain merits and some shortcomings. For example, latex particles easily agglutinate in the presence of binding components. However, in applications where the stability of the conjugate is critical to avoid false positives, the agglutination of latex particles can be a problem. Similarly, enzyme labels offer high sensitivity, however, enzymes sometimes produce an amorphous visual product, which may diffuse across the test strip, and render the results ambiguous. Enzyme labels can also suffer from stability problems.

The advantages of gold labels include the ease of manufacturing uniform-sized particles and the ability to easily bind proteins to these particles. In addition, they also exhibit long-term stability in both liquid and dried forms. Gold labels have sensitivities comparable to enzyme-linked immunosorbent assay (ELISA). The main drawback of colloidal gold 
particles is that quantifiable measurements are difficult. To address these problems, we utilized quantum dots as labels as part of the lateral flow test strip platform. Quantum dots are excited with a single light source (because QDs are excited with UV light at 365-390 $\mathrm{nm}$ ), and emit spectrally in a narrow region. This results in significant improvement in the capabilities of the test strips, and reduces the cost of the reader.

We have utilized quantum dot-based labeling technology to fabricate lateral flow test strips for the quantitative multiplexed detection of biomarkers from serum, urine, and other sample matrices. An optical test strip reader to measure, analyze, and archive the data has been developed. The reader is battery powered and can be used in doctors' offices, as well as for field-based applications. A photograph of the cassette reader and the test strip cassettes is shown in Figure 5.

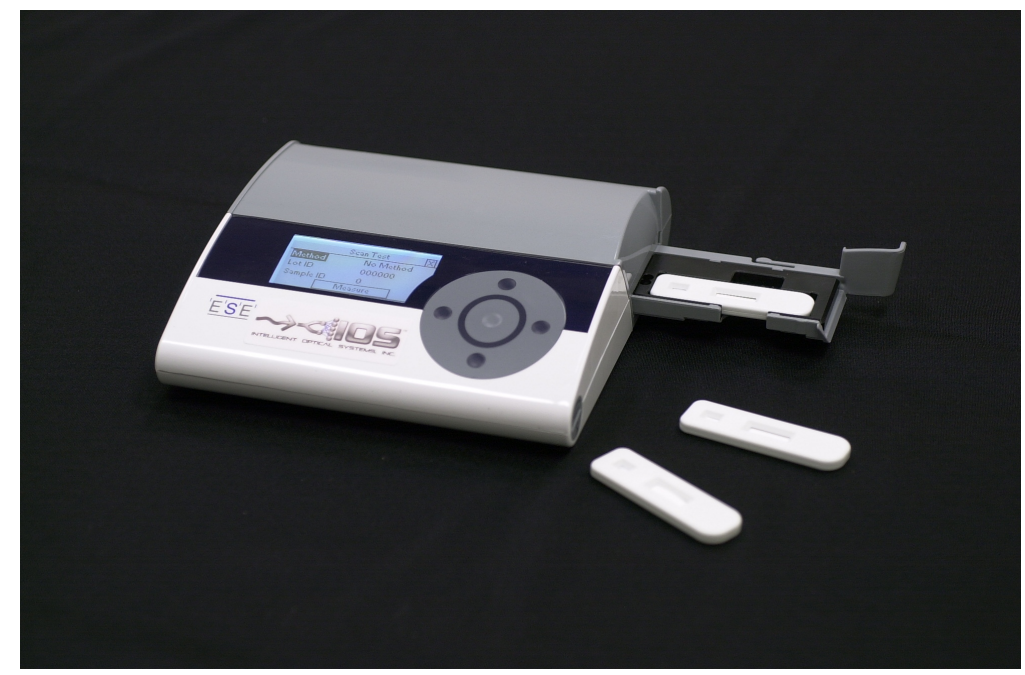

Figure 5 Quantum dot label-based lateral flow test strip reader.

\section{RESULTS}

\subsection{Imaging ellipsometer assay platform testing}

To demonstrate the feasibility of detection, we selected the binding of IgG to Protein G as a model system. Epoxy derivatized microscope glass slides from Telechem International were purchased. Purified recombinant Protein $G$ (Catalog \# 21193) and Immunopure goat anti-mouse $\operatorname{IgG}(\mathrm{H}+\mathrm{L})$ (Catalog \# 31160) were purchased from Pierce Biotechnology Inc (Rockford, IL). Protein G, Bovine Serum Albumin (BSA) and goat anti-mouse IgG were spotted on an epoxy derivatized microscope glass slide using a microspotter. The proteins were spotted as four separate grids. Each grid has six rows and eight spots in each row. Protein $\mathrm{G}$ was spotted on the first and second rows, the third and fourth rows were spotted with BSA, and the fifth and sixth rows contained goat anti-mouse IgG. BSA and goat anti-mouse IgG function as negative controls. The binding to the Protein G spots were studied.

The prepared arrays were washed three times with PBS buffer, and were blocked with $1 \%$ BSA in PBS buffer. The array was placed over the prototype reader and a retardance image of the array was taken. The retardance image was calculated using mini-ellipsometer LabVIEW software. Two images (at $+5^{\circ}$ and $-5^{\circ}$ analyzer position) were taken. These two images were used to calculate the retardance of the array (Figure 6a). Next, the microarray was incubated with goat anti-mouse IgG for ten minutes. The sample was washed in PBS buffer, and the retardance image was then taken (Figure $6 b)$. The surface plots of the same images are shown in Figures $7 \mathrm{a}$ and $7 \mathrm{~b}$, respectively. 


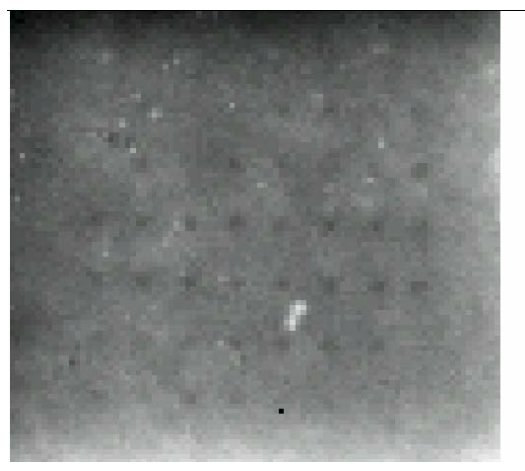

(a)

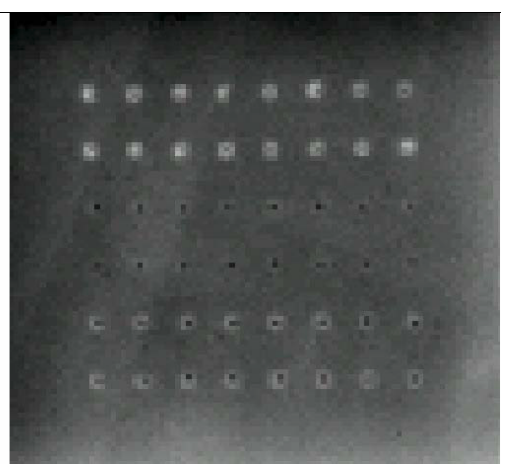

(b)

Figure 6 Retardance images of the biochip taken by the benchtop prototype: (a) before binding; and (b) after binding goat anti-mouse IgG.
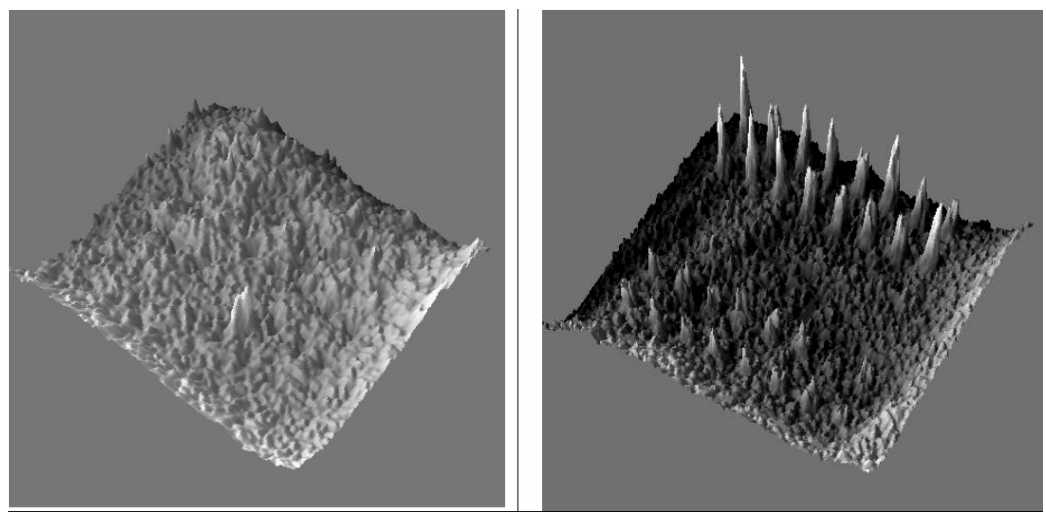

Figure 7 Surface plots of retardance images taken using the prototype reader: (a) before binding; and (b) after binding goat anti-mouse IgG.

For a validation experiment, the microarray was incubated with Hilyte Fluor ${ }^{\mathrm{TM}} 647$ labeled goat anti-mouse IgG, and was washed with PBS. The fluorescence intensities from the spots were measured with a fluorescent scanner (Figure 8).

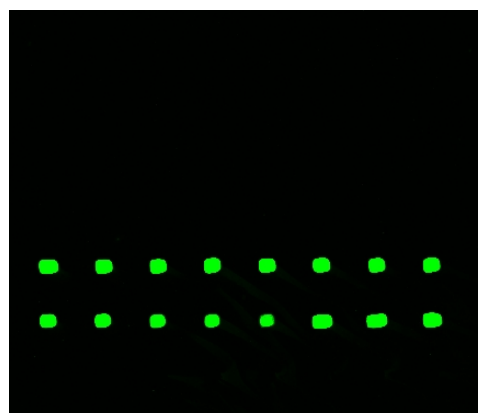

Figure 8 The fluorescent image of the microarray used for validation experiments. The first rows that contain Protein G bind strongly to fluorescent labeled goat anti-mouse IgG.

\subsection{TBI diagnosis}

One in every four combat casualties is due to battlefield head injuries. Clinically, head injuries result in cognitive deficits, and in impaired memory, judgment, and perception. The closed head injuries caused by impacts from improvised explosive devices (IEDs) and rocket propelled grenades are a major concern, because they are difficult to detect. The early detection of these head injuries in battlefield settings is critical to improving the treatment and therapy of injured personnel. Additionally, the detection device must be simple to use; the test must be based on easy to collect samples (e.g., blood, serum, urine, or saliva); and the test must be sensitive and specific. Recent studies have resulted in 
the discovery of several promising biomarkers, such as S100B, isoprostane, glial fibrillary acidic protein (GFAP), and neuron specific enolase (NSE), that could be useful in diagnosing brain injury.

Starting from 10 hours, serum samples were collected every 12 hours until 136 hours after TBI insult, from adult TBI patients $(\mathrm{n}=20$, age 21-74 years) with a Glasgow Coma Scale score $\leq 8$. Biomarkers were measured and the results showed that S100B, NSE, and GFAP were elevated in TBI patients, and reached peak concentrations at $40 \mathrm{~h}, 50 \mathrm{~h}$, and $40 \mathrm{~h}$ after injury, respectively. The GFAP levels reached base line values by $64 \mathrm{~h}$ after injury. A 2.64, 12.8, and 56.3 fold increase in NSE, S100B and GFAP respectively were observed between TBI and controls. F2-isoprostane was not significantly increased in TBI patients. More tests and data analysis are underway to relate the biomarkers to the severity and outcome of TBI by using patients with mild/moderate TBI and patients with peripheral injuries as controls.

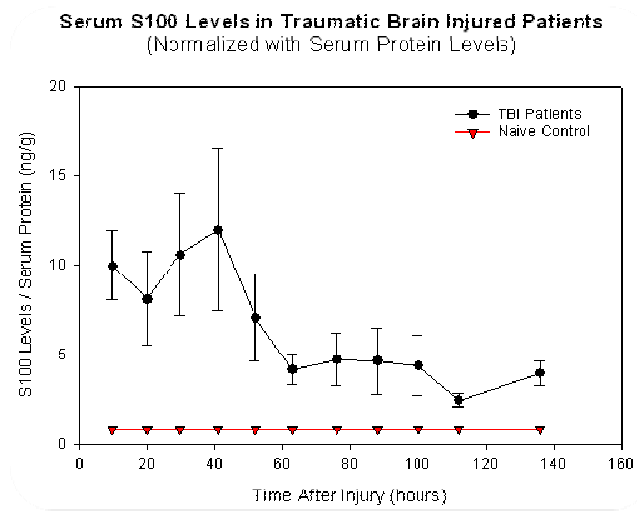

Serum GFAP Levels in Traum atic Brain Injured Patients (Normalized with Serum Protein Levels)

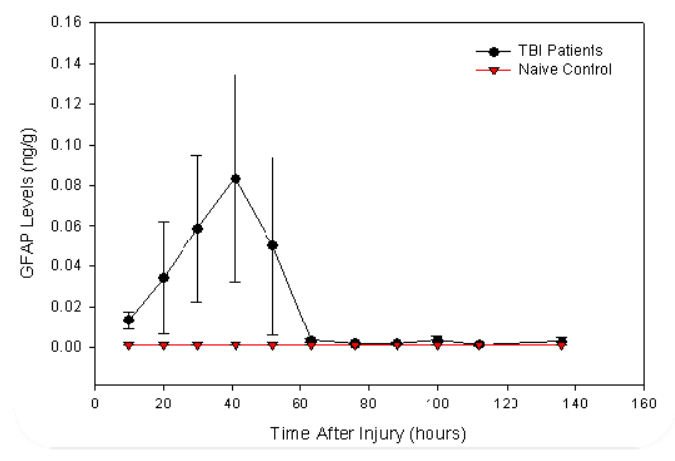

Serum NSE Levels in Traum atic Brain Injured Patients (Normalized with Serum Protein Levels)

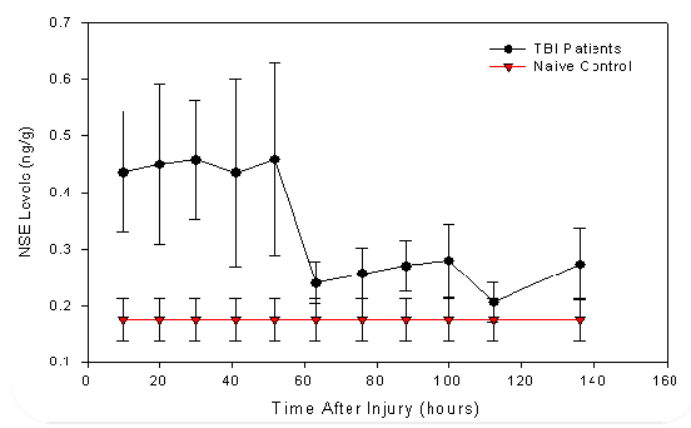

Serum F2-Isoprostane Levels in Traumatic Brain Injured Patients (Nomalized with Serurr Proteirı Levels)'

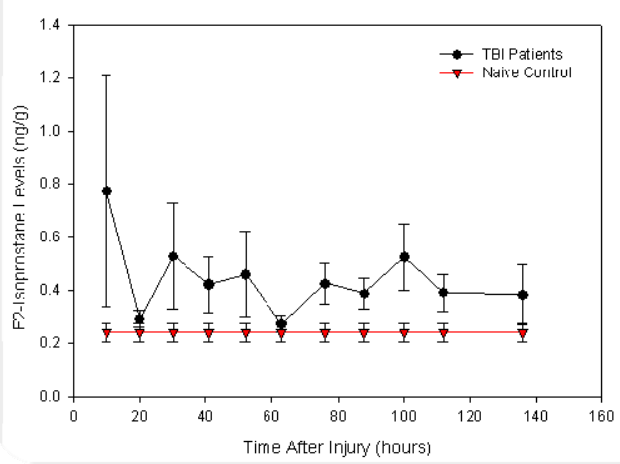

Figure 9 Time course of measured TBI biomarkers in patients. Results show a strong elevation in the concentrations of S100B, NSE and GFAP in TBI patients.

\section{CONCLUSIONS}

Fieldable biomarker detection technologies can have a significant impact on field medicine, and can lead to the timely and accurate diagnosis of conditions such as traumatic brain injury. Both the lateral flow technology and the microfluidic technology can be adapted to achieve robust and accurate devices. The clinical data suggests that protein-based biomarkers are elevated after TBI, and can be used to develop a diagnostic tool.

\section{ACKNOWLEDGMENTS}

This work was funded by support from an Army SBIR contract (Contract \# W81XWH07-C-0031) and an Air Force SBIR Contract (FA8650-07-C-6757). The author is indebted to project technical monitors Dr. Madhusoodana Nambiar and Dr. Camilla Mauzy for their suggestions and support in the development of this work. 


\section{REFERENCES}

[1] Kempen, L.U., "Imaging apparatus and method," U.S. Patent No. 6,594,011 (7/15/2003).

[2] Chipman, R.A. [Handbook of Optics] McGraw-Hill, Chapter 22, Polarimetry (1995).

[3] Maxwell-Garnett, J.C., "Colours in metal glasses and in metallic films," Philos. Trans. R. Soc. Lond. A 203, 385 (1904).

[4] Maxwell-Garnett, J.C., "Colours in metal glasses and in metallic films," Philos. Trans. R. Soc. Lond. A 205, 237 (1906). 